Pacific Journal of Mathematic 


\title{
DECOMPOSITIONS OF INJECTIVE MODULES
}

\author{
R. B. WARFIELD, JR.
}

The main results of this paper concern decompositions of an injective module, either as a direct sum of submodules or as the injective envelope of a direct sum of injective submodules. This second kind of decomposition can be regarded as an ordinary direct sum (coproduct) in a suitable Abelian category - the spectral category of the ring. The results are therefore put in the context of Abelian categories, and the main result is that in an Abelian category satisfying axiom $A b-5$ and with infinite direct sums, any two direct sum decompositions of an injective object have isomorphic refinements.

This is particularly strong if decompositions into indecomposable injectives exist, and it enables one to classify the injective modules over a valuation ring. Such strong results as this are not available for more general classes of modules, but in $\S 3$ the methods of Crawley and Jónsson are exploited to obtain results in certain cases; for example, for modules which are direct sums of countably generated modules. The Crawley-Jónsson results are put into the context of category theory and an example is given (involving relatively injective modules) to show how the hypotheses can be weakened by working in a subcategory of the category of $R$-modules.

A remark should be made on the types of decompositions we consider for injective modules in $\S 2$. For injective modules over Noetherian rings, ordinary direct sums yield excellent results, due primarily to Matlis [7]. In contrast, Faith and Walker [2] have shown that if $R$ is a non-Noetherian ring, there does not exist any set of injective modules such that any injective module can be imbedded in a direct sum of modules isomorphic to members of this set. In the spectral category, however, reasonable decompositions always exist (Theorem 2 below). The spectral category was introduced by Gabriel and Oberst in [4] and exploited in [10]. The author is indebted to Professor J. E. Roos for pointing out the connection between these two papers and the work reported here.

We do not consider Cartesian product decompositions of injective modules, since product decompositions simply do not have the necessary uniqueness properties. For an example let $Q$ and $Z$ denote the additive groups of rationals and integers, respectively, and $(Q / Z)_{p}$ the $p$-primary component of $Q / Z$. Then

$$
\Pi_{p}(Q / Z)_{p} \cong Q \times \Pi_{p}(Q / Z)_{p}
$$

so that we have two product decompositions of an injective $Z$-module 
into indecomposables, and these decompositions are in no sense equivalent.

1. Decompositions in Abelian categories. We will work in Abelian categories satisfying the usual axioms (as in MacLane [6, pp. 248-257]) together with the following three conditions:

(i) The set-theoretic axiom [6, p. 253] that for each object $A$ there is a set of subobjects, such that any subobject is equivalent to a member of this set.

(ii) We assume that arbitrary direct sums (coproducts, cocartesian products) exist.

(iii) We assume the axiom $\mathrm{Ab}-5$ in the following form: if $A_{i}$ is direct family of subobjects of an object $C$, and $B$ is a subobject of $C$, then

$$
\left(\bigcup A_{i}\right) \cap B=\bigcup\left(A_{i} \cap B\right) \text {. }
$$

In clarification of condition (iii), we should remark that if $A_{i}(i \in I)$ is a family of subobjects of an object $C$ then their injection homomorphisms induce a unique homomorphism from their direct sum (coproduct) into $C$, and the image of this homomorphism is the union (or join) of the $A_{i}$, and is denoted $\cup A_{i}$. Similarly, if $A$ and $B$ are subobjects, then $A \cap B$ is the kernel of the natural homomorphism $C \rightarrow C / A \oplus C / B$.

Definition. An Abelian category satisfying the conditions (i), (ii), (iii) above will be called a reasonable Abelian category.

In general one can work with direct sums in a reasonable Abelian category just as one would with direct sums of modules. The notion of a decomposition of an object into a direct sum of subobjects, $A=$ $\bigoplus_{i \in I} A_{i}$, has the obvious interpretation, and two decompositions are isomorphic if the summands are isomorphic in pairs. If also $A=$ $\bigoplus_{j \in J} B_{j}$, we say the second decomposition is a refinement of the first if there is a surjective map $\phi: J \rightarrow I$ such that $B_{j} \subseteq A_{\phi(j)}$, from which it follows that the induced morphism

$$
\bigoplus_{\phi(j)=i} B_{j} \longrightarrow A_{i}
$$

is an isomorphism. The direct sum of two objects $A$ and $B$ will be written $A \oplus B$, and if $A$ and $B$ are subobjects of $C$ with $A \cap B=0$, then their join is isomorphic to $A \oplus B$ and will be denoted $A \oplus B$.

Two more remarks should be made: First, if $C=A \oplus B$, and $D \leqq C$, then $C=D \oplus B$ if and only if $\pi_{A} \circ \dot{\phi}_{D}$ is an isomorphism. (Here $\pi_{A}$ is the natural projection $C \rightarrow A$, and $\phi_{D}$ the natural injection $D \rightarrow C$.) Secondly, if $C=A \oplus B$ and $A \subseteq D$ (another subobject), then 
$A$ is a summand of $D$ and $D=A \oplus(D \cap B)$.

We recall that a subobject $L \subseteq A$ is essential if for any subobject $S \subseteq A$, if $S \neq 0$, then $S \cap L \neq 0$. If $D$ is an injective subobject of $A$ and $D$ is essential, then $D=A$.

Lemma 1. If $A=\bigoplus_{i \in I} A_{i}$ (in any reasonable Abelian category) and $S$ is a subobject of $A$, then $S$ is essential in $A$ if and only if $S \cap A_{i}$ is essential in each $A_{i}$.

Proof. That the condition is necessary is clear. Conversely, suppose that $B \neq 0$ is a subobject of $A$. By (iii), there is a finite subset $I^{*} \subseteq I$ with $B \cap\left(\bigoplus_{i \in I^{*}} A_{i}\right) \neq 0$. Therefore, to show that our condition implies $S$ is essential, we need only show that $S$ is essential in finite subsums. By iteration, we need only show that if $S \subseteq C_{1} \oplus C_{2}$ and $S \cap C_{i}$ is essential in $C_{i}$, then $S$ is essential in $C_{1} \oplus C_{2}$. Let $B \neq 0$ be a subobject of $C_{1} \oplus C_{2}$ and let $\pi_{1}$ and $\pi_{2}$ be the projections to $C_{1}$ and $C_{2}$. Let $\dot{\phi}: B \rightarrow C_{1}$ be the restriction of $\pi_{1}$ to $B$. If $B \cong C_{2}$ then clearly $B \cap S \neq 0$, so we assume this is not the case, in which case $\operatorname{im}(\phi) \neq 0$. Hence $\operatorname{im}(\phi) \cap S \neq 0$, so we let

$$
B^{\prime}=\phi^{-1}(\operatorname{im}(\phi) \cap S) \text {. }
$$

Since $B^{\prime} \neq 0$, we need only show $B^{\prime} \cap S \neq 0$. Let $\psi$ be the restriction of $\pi_{2}$ to $B^{\prime}$. By the same argument as before, we may assume that $B^{\prime}$ is not contained in $C_{1}$, so that $\operatorname{im}(\psi) \neq 0$. We let

$$
B^{\prime \prime}=\psi^{-1}(\mathrm{im}(\psi) \cap S)
$$

and it is clear that $B^{\prime \prime} \neq 0, B^{\prime \prime} \subseteq S$, so that $B \cap S \neq 0$ as desired.

LEMMA 2. (The exchange property) If $M$ is an object in a reasonable Abelian category and $D$ is an injective subobject, and $M=$ $\bigoplus_{i \in I} M_{i}$, then there subobjects $M_{i}^{\prime} \subseteq M_{i}$, so that $M=D \oplus\left(\bigoplus_{i \in I} M_{i}^{\prime}\right)$.

Proof. Let $S \subseteq M$ be a subobject chosen maximal with respect to the following properties:

(1) $S=\bigoplus_{i \in I} S \cap M_{i}$

(2) $S \cap D=0$.

To show such an $S$ exists note that (i) enables us to apply Zorn's lemma and (iii) guarantees that an ascending union of subobjects satisfying (2) still satisfies (2). Let $M_{i}^{\prime}=S \cap M_{i}$. Then we claim $M=$ $D \oplus\left(\bigoplus_{i \in I} M_{i}^{\prime}\right)$.

Let $\phi$ be the natural map from $M$ to $M / S$, and let $\psi$ be the restriction of $\phi$ to $D$. Condition (2) above implies that $\psi$ is a monomorphism, so $\operatorname{im}(\psi)$ is an injective subobject of $M / S$. We need only 
show that $\operatorname{im}(\psi)$ is essential, which is an easy consequence of Lemma 1.

Lemma 3. Let $D$ be an injective object in a reasonable Abelian category, and $A$ a subobject of $D$. Then there is an injective subobject $E$ of $D$ with $A \subseteq E$, and $A$ essential in $E$.

Proof. By axiom (i), we can choose subobjects $E$ and $S$ of $D$ such that $E$ is maximal with respect to the property that $A$ is essential in $E$, and $S$ is maximal with respect to the property that $S \cap A=0$. Let $\phi: D \rightarrow D / S$ be the natural homomorphism. If $\phi^{\prime}$ is the restriction of $\phi$ to $E$, then $\phi^{\prime}$ is a monomorphism. We therefore have a homomorphism carrying im $\left(\phi^{\prime}\right)$ back to $E$, and since $D$ is injective, this extends to a homomorphism $\psi: D / S \rightarrow D$. Since $E \leqq \operatorname{im}(\psi)$ and $A$ is essential in im $(\psi)$, we have $E=\operatorname{im}(\psi) . E$ is therefore a summand of $D$, with projection $\psi \circ \phi$, and hence $E$ is injective.

Theorem 1. If $D$ is an injective object in a reasonable Abelian category, then any two direct sum decompositions of $D$ have isomorphic refinements.

Proof. We will consider two decompositions of $D$ and we assume the summands are well ordered, so that we can take ordinal numbers as our indices and write

$$
D=\bigoplus_{i<N} A_{i}=\bigoplus_{j<M} B_{j} .
$$

We will construct subobjects $C_{i j}(i<N, j<M)$ of $D$, such that $D=$ $\bigoplus_{i j} C_{i j}, A_{i} \cong \bigoplus_{j<M} C_{i j}, B_{j} \cong \bigoplus_{i<N} C_{i j}$. The construction will be carried out by induction on pairs of indices $(n, j)$. For each pair $(n, j)$ we will want the following statements to hold:

$1(n, j)$. For $i<n$ there are subobjects $A_{i j} \leqq A_{i}$ and for $i<n$, $k<j$, the subobjects $C_{i k}$ have been constructed.

$2(n, j) . \quad \bigoplus_{i<n} A_{i}=\left(\bigoplus_{i<n} A_{i j}\right) \bigoplus\left(\bigoplus_{i<n} \bigoplus_{k<j} C_{i k}\right)$.

$3(n, j) . \quad\left(\bigoplus_{k<j} B_{k}\right) \cap\left(\bigoplus_{i<n} \bigoplus_{k<j} C_{i k}\right)$ is essential in both $\left(\bigoplus_{k<j} B_{k}\right) \cap$ $\left(\bigoplus_{i<n} A_{i}\right)$ and $\bigoplus_{i<n} \bigoplus_{k<j} C_{i k}$.

Suppose that the $C_{i j}$ have been constructed so that all of the statements $1(n, j), 2(n, j), 3(n, j)$ hold for $n \leqq N, j \leqq M$. Then $2(N, M)$ and $3(N, M)$ together imply that

$$
D=\bigoplus_{i<N} \bigoplus_{j<M} C_{i j}
$$

and hence by $2(n, M)$ and $2(n+1, M)$

$$
\begin{aligned}
\bigoplus_{i<n} A_{i} & =\bigoplus_{i<n} \bigoplus_{j<M} C_{i j} \\
\bigoplus_{i<n+1} A_{i} & =\bigoplus_{i<n+1} \bigoplus_{j<M} C_{i j}
\end{aligned}
$$


and comparing these two statements, we conclude that

$$
A_{n} \cong \bigoplus_{j<M} C_{n j}
$$

since two complements to the same summand are isomorphic.

We now need to note that if $D$ is injective and $A, B$ are summands of $D$ with $A \cap B$ essential in both $A$ and $B$, and if $D=A \oplus C$, then $D=B \oplus C$ also, and in particular, $A \cong B$. To apply this, we note that for any index $j$,

$$
\left(\bigoplus_{k<j} B_{k}\right) \cap\left(\bigoplus_{k<j} \bigoplus_{i<N} C_{i k}\right)
$$

is essential in both, so that the first summand may be replaced by the second. Doing this, and then applying the same remark for the index $j+1$, we obtain the following expressions

$$
\begin{aligned}
D & =\left(\bigoplus_{k<j} \bigoplus_{i<N} C_{i k}\right) \oplus B_{j} \oplus\left(\bigoplus_{j<k} B_{k}\right) \\
& =\left(\bigoplus_{k<j} \bigoplus_{i<N} C_{i k}\right) \oplus\left(\bigoplus_{i<N} C_{i j}\right) \oplus\left(\bigoplus_{j<k} B_{k}\right) .
\end{aligned}
$$

Comparing middle terms, we obtain as before

$$
B_{j} \cong \bigoplus_{i<N} C_{i j}
$$

as desired.

We now complete the proof by carrying out the construction of the subobjects $C_{i j}$ in the required way. We first use induction on the index $j$. The construction is completed for the index $j$ if subobjects $A_{i j}$ have been constructed for all $i<N$, and subobjects $C_{i k}$ for $i<N$, $k<j$, so that the statements $1(i, k), 2(i, k), 3(i, k)$ holds for all $i \leqq N$, $k \leqq j$. Suppose, now, that the construction has been completed for all indices $k, k<j$, and that $j$ is a limit ordinal. Conditions $3(N, k)$ and $3(N, k+1)$ show that $C_{i k}$ is isomorphic to a summand of $B_{k}$, so $\bigoplus_{k<j} C_{i k}$ is isomorphic to a summand of $D$, and hence is injective. $2(i, k)$ and $2(i+1, k)$ imply that the projection of $\bigoplus_{k<j} C_{i k}$ into $A_{i}$ is a monomorphism, so $\bigoplus_{k<j} C_{i k}$ is isomorphic to a summand of $A_{i}$. Summing over $i$, we find that $\bigoplus_{i<n} \bigoplus_{k<j} C_{i k}$ is isomorphic to a summand of $D$ (for all $n<N$ ) and hence is also injective. We can therefore apply the exchange property (Lemma 2) for this subobject and obtain subobjects $A_{i j}(i<N)$ such that

$$
D=\left(\bigoplus_{i<N} \bigoplus_{k<j} C_{i k}\right) \oplus\left(\bigoplus_{i<N} A_{i j}\right) .
$$

This is not quite good enough, but if we do this inductively for each index $n$ in turn, we can also guarantee that

$$
\bigoplus_{i<n} A_{i}=\left(\bigoplus_{i<n} \bigoplus_{k<j} C_{i k}\right) \bigoplus\left(\bigoplus_{i<n} A_{i j}\right)
$$

for all $n<N$, so that for all $n<N, 2(n, j)$ will hold. Condition 
$3(n, j)$ is immediate (since if $K_{i}$ is an ascending family of subobjects of an object $K$ and $L_{i}$ is an ascending family of subobjects with $L_{i} \subseteq K_{i}, L_{i}$ essential in $K_{i}$, then axiom (iii) implies that $\bigcup L_{i}$ is essential in $\left.\cup K_{i}\right)$. This completes the induction at a limit ordinal.

To complete the proof, we must show that if the constuction has been completed for an index $j$, it can be completed for $j+1$. We do this by induction on $n$, establishing $1(n, j+1), 2(n, j+1)$, $3(n, j+1)$. Suppose that $n$ is a limit ordinal, so that the $A_{i, j+1}$ are constructed for all $i<n$ and $C_{i k}$ for $i<n, k<j+1$. Applying $2(i, j+1)$ for all $i<n$ and taking an ascending union we see that $2(n, j+1)$ is immediate, and $3(n, j+1)$ is also immediate because (as we saw at the end of the previous paragraph) ascending unions preserve "essentialness".

We must show, finally, that if we can carry out our construction so that $1(n, j+1), 2(n, j+1), 3(n, j+1)$ (and also $1(n+1, j), 2(n+1, j)$, $3(n+1, j))$ hold, then we can construct $A_{n, j+1}, C_{n, j}$ so that $1(n+1, j+1)$, $2(n+1, j+1), 3(n+1, j+1)$ also hold. We have

$$
\bigoplus_{i<n} A_{i}=\left(\bigoplus_{i<n} A_{i, j+1}\right) \bigoplus\left(\bigoplus_{i<n} \bigoplus_{k<j+1} C_{i k}\right)
$$

and the equation remains true if we add $A_{n}$ on the left and

$$
A_{n j} \oplus\left(\bigoplus_{k<j} C_{n k}\right)
$$

on the right. We choose $C_{n j}$ in $\bigoplus_{i<n+1} A_{i}$ to be maximal with respect to the properties that

(1) $C_{n j} \cap\left[\left(\bigoplus_{i<n} A_{i}\right) \oplus\left(\bigoplus_{k<j} C_{n k}\right)\right]=0$

(2) $\left(\bigoplus_{i<n+1} \bigoplus_{k<j+1} C_{i k}\right) \cap\left(\bigoplus_{k<j+1} B_{k}\right)^{\prime}$

is essential in $\bigoplus_{i<n+1} \bigoplus_{k<j+1} C_{i k}$. It is clear from Lemma 3 that $C_{n j}$ is injective. $3(n+1, j+1)$ is clearly satisfied, and applying the exchange property (for the summand $\left(\bigoplus_{i<n} A_{i}\right) \bigoplus\left(\bigoplus_{k<j+1} C_{n k}\right)$ in $\left.\bigoplus_{i<n+1} A_{i}\right)$ we can find a complement $A_{n, j+1}$ so that $2(n+1, j+1)$ also holds, thus completing the induction.

\section{Applications.}

Corollary 1.1. Any two direct sum decompositions of an injective $R$-module have isomorphic refinements.

To obtain more useful results, we consider another sort of decomposition.

Theorem 2. Let $R$ be an associative ring with 1. Then any injective $R$-module is the injective envelope of a direct sum of injective submodules isomorphic to $E(R / I)$ (for varying $I$ ) where $R / I$ is a cyclic left $R$-module and $E(R / I)$ denotes the injective envelope of $R / I$. 
Proof. For injective envelopes see [6, p. 102]. We choose a subset $S$ of $D$ such that

(1) the elements of $S$ are nonzero,

(2) the elements of $S$ are independent (that is, the submodule generated by $S$ is the direct sum of the cyclic modules [x], generated by the elements $x$ in $S$ ),

(3) $S$ is maximal with respect to properties (1) and (2). We now let $B$ be an injective envelope in $D$ of the submodule generated by $S$. $B$ is the injective envelope of a direct sum of cyclic modules, and also, by breaking the process into two stages, the injective envelope of a direct sum of injective submodules of the form $E(R / I)$. It is easy to see that $B=D$.

THEOREM 3. The following conditions on an associative ring $R$ with 1 are equivalent:

(i) If $I$ is a left ideal of $R$, then either $I$ is irreducible or there are left ideals $A, B$, different from $I$, such that $A$ is irreducibe and $I=A \cap B$.

(ii) Any injective left $R$-module has a nonzero indecomposable summand.

(iii) Any injective left $R$-module is the injective envelope of a direct sum of indecomposable injective $R$-modules.

Proof. Much of this is due to Matlis [7]. Suppose that $E$ is the injective envelope of a cyclic submodule $[x]$ and $E=E_{1} \oplus E_{2}$ where both $E_{1}$ and $E_{2}$ are nonzero. If in this decomposition $x=x_{1}+x_{2}$, then it is easy to see that $E_{i}$ is the injective envelope of $\left[x_{i}\right]$ and $o(x)=o\left(x_{1}\right) \cap o\left(x_{2}\right)$, where $o(x)$, and $o\left(x_{i}\right)$ are the order ideals of $x$ and $x_{i}$ respectively. Further, we see that $o(x) \neq o\left(x_{i}\right)$ for either $i$. We conclude that $E(R / I)$ is indecomposable if and only if $I$ is an irreducible ideal, and $E(R / I)$ has an indecomposable summand if and only if $I$ satisfies the conclusion of condition (i) above. Since any injective $R$-module has a summand of the form $E(R / I)$ for some left ideal $I$, the equivalence of (i) and (ii) is now clear. Clearly (iii) implies (ii) and the proof of the converse is essentially the same as the proof of Theorem 2.

Definition. Let $R$ be an associative ring with unit. $\mathscr{I}(R)$ is the category whose objects are injective $R$-modules with morphisms defined by

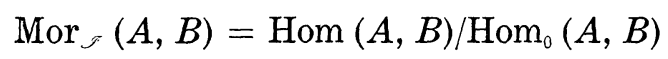


where $\operatorname{Hom}_{0}(A, B)$ is the subgroup of $R$-homomorphisms whose kernel is essential in $A$.

THEOREM 4. $\mathscr{I}(R)$ is a reasonable Abelian category in which all short exact sequences split. Elements of $\mathscr{G}(R)$ are isomorphic in $\mathscr{J}(R)$ if and only if they are isomorphic as modules, and if $A_{i}(i \in I)$ is a family of injective modules, then their direct sum in the category $\mathscr{I}(R)$ can be identified with the injective envelope of their module direct sum.

Proof. Most of the proof consists of trivial verifications which will be omitted. We take it as obvious that $\mathscr{F}(R)$ is an additive category satisfying the set theoretic axiom (i). The direct sum of two objects in $\mathscr{J}(R)$ in just their direct sum as $R$-modules. If $f: A \rightarrow B, g: B \rightarrow A$ establish an isomorphism in $\mathscr{J}(R)$ between $A$ and $B$ then $g \circ f$ restricts to the identity function on some essential submodule of $A$ so $g \circ f$ is an automorphism of $A$, and similarly $f \circ g$ is an automorphism of $B$, so $A$ and $B$ are isomorphic as modules.

Let us identify the kernels and cokernels. Let $f \in \operatorname{Hom}(A, B)$ and let $[f]$ denote the correspoding element of $\operatorname{Mor}_{\mathcal{F}}(A, B)$. If $K$ is the kernel of $f$, and $E$ is an injective envelope of $K$ in $A$, then $E$ is a kernel for $[f]$. We can write $A=E \oplus F$ (in $\mathscr{J}(R)$ or as $R$-modules). Let $\pi$ be the projection of $A$ onto $F$ and $f^{\prime}$ the element of $\operatorname{Hom}(F, B)$ induced by $f$. Then $[f]=\left[f^{\prime}\right] \circ[\pi]$ since $f$ and $f^{\prime} \circ \pi$ agree on the essential submodule $K+F$. This is the factorization of $[f]$ into the product of an epimorphism and a monomorphism required in an Abelian category. Finally, $f^{\prime}(F)$ is a summand of $B$ so $B / f^{\prime}(F)$ is injective, and if $\phi$ is the natural homomorphism from $B$ to $B / f^{\prime}(F)$ then $[\dot{\phi}]$ is a cokernel for $[f]$. This shows that $\mathscr{J}(R)$ is an Abelian category, so all that remains is to check conditions (ii) and (iii).

The statement on direct sums is a consequence of Lemma 1. For (iii), note that if we have a directed family of $\mathscr{J}(R)$-subobjects of an injective module $C$, we can choose representative submodules $A_{i}(i \in I)$ for these subobjects so that the family $A_{i}$ is a directed family in the usual sense. $\bigcup A_{i}$ (in the category $\mathscr{J}(R)$ ) can be identified with any injective envelope of the ordinary union of the $A_{i}$. Similarly, $A \cap B$ (in the category $\mathscr{J}(R)$ ) can be identified with any injective envelope of the ordinary intersection. Both of these are well defined in $\mathscr{F}(R)$. To prove that

$$
\left(\bigcup A_{i}\right) \cap B=\bigcup\left(A_{i} \cap B\right)
$$

in the category $\mathscr{J}(R)$, note that the term on the left is any injective 
envelope of $B \cap K K$ where is an injective envelope of the ordinary union of the $A_{i}$. If this ordinary union is denoted by $A$, then since $A$ is essential in $K$, the term on the left represents an injective envelope of $A \cap B$. Similarly, the term on the right represents an injective envelope of $A \cap B$, from which the result follows.

We should remark that this theorem carries over to any reasonable Abelian category in which injective envelopes exist.

CoRollary 4.1. Any two representations of an injective module as the injective envelope of a direct sum of injective submodules have isomorphic refinements.

Corollary 4.2. Let $M$ be an injective module which is the injective envelope of a direct sum of indecomposable injective submodules $E_{i}(i \in I)$. Then any two such decompositions are isomorphic, and furthermore, if $N$ is an injective submodule of $M$, there is a subset $J \subseteq I$ such that $M=N \oplus E\left(\bigoplus_{i \in j} E_{i}\right)$.

The second half of this corollary follows from Lemma 2, in the category $\mathscr{F}(R)$. Theorem 3 gives conditions to which this corollary applies. Another such condition, in terms of transfinite Krull dimension, is given by Gabriel [3, pp. 382, 386]. A similar result in [10].

We recall that a commutative ring $R$ is a valuation ring if it is an integral domain and for any two nonzero elements $r$ and $s$ of $R$, either $r$ divides $s$ or $s$ divides $r$. It follows that if $I$ and $J$ are ideals, either $I \subseteq J$ or $J \subseteq I$. Hence any ideal is irreducible, and it follows that the injective envelope of a cyclic module, $E(R / I)$, is always indecomposable. One can show further [8] that $E(R / I) \cong E(R / J)$ if and only if there are nonzero elements $r, s$ of $R$ such that $r I=s J$, (or equivalently, $I$ and $J$ are isomorphic as modules). Applying Theorem 2 and Corollary 4.2 , we obtain the following.

COROLlary 4.3. An injective module over a valuation ring is the injective envelope of a direct sum of indecomposable injective modules, and any two such decompositions are isomorphic. An injective module is indecomposable if and only if it is of the form $E(R / I)$, and $E(R / I) \cong E(R / J)$ if and only if $I \cong J$.

Other consequences, not directly involving injective modules, also follow from these results. For the following, let $R$ be a commutative integral domain. We recall that a torsion-free module is reduced if it has no nonzero injective summand, or equivalently if no nonzero element is divisible by all elements of $R$. If $A$ is a submodule of a module $B, A$ is $R D$-pure in $B$ if for all $r \in R, r A=A \cap r B$. ( $R D$ here 
tands for"relatively divisible".) A module $M$ is $R D$-injective if it is a summand of any module which contains it as an $R D$-pure submodule. By [12, Corollary 2] the functor $\operatorname{Hom}(Q / R, \cdot)$ gives a category isomorphism between the category of torsion, injective $R$-modules and the category of reduced, torsion-free $R D$-injective $R$-modules. (Here, $Q$ is the quotient field of $R$. This result is a corollary of the category isomorphism theorem of Matlis [9, Th. 3.4].) We also have a notion of $R D$-injective envelope for this theory, and we can actually write down an explicit formula. If $A$ is a reduced torsion-free module, its $R D$ injective envelope is $\operatorname{Hom}(Q / R, E(A \otimes(Q / R)))$, where $E(M)$ is the ordinary injective envelope of $M$. All of the previous results for injective modules now carry over because of the category isomorphism theorem mentioned above, but we content ourselves with a version of Corollaries 4.2 and 4.3 .

COROLlary 4.4. If $M$ is a reduced torsion-free RD-injective module over an integral domain, and if $M$ is the RD-injective envelope of a direct sum of indecomposable RD-injective modules, then any two such decompositions of $M$ are isomorphic. If the domain is a valuation ring, any reduced torsion-free $R D$-injective module is the RD-injective envelope of a direct sum of ideals, and any two such representations are isomorphic.

The only additional remark needed to complete the proof of this is that if $R$ is a valuation ring, the $R D$-injective envelope of an ideal $I, I \neq R$, is $\operatorname{Hom}(Q / R, E(R / I))$ since $R / I$ is essential in $Q / I$, and there is a natural isomorphism $Q / I \cong(Q / R) \otimes I$.

3. The Crawley-Jónsson theorems. We wish to review here some important results on direct sum decompositions due to Crawley and Jonsson [1] and to place them in the context of Abelian categories. We should remark that Crawley and Jonsson work with general algebraic systems, and their results are valid in many categories that are not even additive, so that our results do not contain theirs. Our proofs are valid in somewhat more general categories than reasonable Abelian categories, however-in particular in any full subcategory which is closed under summands and direct sums (for example, in the category of torsion-free Abelian groups), and the hypotheses are often weakened by restricting to a subcategory.

Definition. An object $D$ in an Abelian category has the exchange property if for any object $A$, if we have

$$
A=D^{\prime} \oplus B=\bigoplus_{i \in I} A_{i}
$$


with $D^{\prime} \cong D$, then there are subobjects $A_{i}^{\prime} \cong A_{i}$ such that

$$
A=D^{\prime} \oplus\left(\bigoplus_{i \in I} A_{i}^{\prime}\right) \text {. }
$$

Similarly, $D$ has the finite exchange property if this conditions holds whenever the set $I$ is finite.

Theorem 5. [1, Th. 4.2]. If $M$ is an object in a reasonable Abelian category and $M=\bigoplus_{i \in I} A_{i}=\bigoplus_{j \in J} B_{j}$ where the sets $I$ and $J$ are countable and the subobjects $A_{\text {; }}$ and $B_{j}$ have the exchange property, then these two decompositions have isomorphic refinements.

The proof is a diagonal argument and we refer to [1, pp. 817-818] for details. The countability hypothesis seems to be essential. It can be removed, however, by placing a countability hypothesis on the summands. Crawley and Jónsson therefore assume their summands are countably generated, and the following definition provides a substitute for this in a general setting.

Definition. An object $D$ in an additive category is small if for any direct sum $A=\bigoplus_{i \in I} A_{i}$, with projections $\pi_{i}$, and any morphism $f: D \rightarrow A$, we have $\pi_{i} \circ f=0$ for all but a finite number of indices $i$. $D$ is $\sigma$-small if it is a countable ascending union of small subjects. $D$ is countably small if for any direct sum $A=\bigoplus_{i \in I} A_{i}$, and $f: D \rightarrow$ $A$, we have $\pi_{i} \circ f=0$ for all but a countable number of indices $i$.

Lemma 4. Let $N$ be a summand of an object $M$ in a reasonable Abelian category such that $M$ is the direct sum of countably small subobjects. Then $N$ is also a direct sum of countably small subobjects.

This is essentially equivalent to [5, Th. 1]. $\sigma$-small can be substituted for countably small, and suitable versions for larger cardinals also are valid.

Lemma 5. Let $M$ be an object in a reasonable Abelian category and $M=\bigoplus_{i \in I} A_{i}=\bigoplus_{j \in J} B_{j}$, where each of the summands is countably small. Then we can decompose $I$ and $J$ into disjoint, countable subsets $I_{\lambda}, J_{\lambda}(\lambda \in \Lambda)$, such that

$$
\bigoplus_{i \in I_{\lambda}} A_{i} \cong \bigoplus_{j \in J_{\lambda}} B_{j}(\lambda \in \Lambda) .
$$

Proof. We outline the proof, which is a straightforward elementary argument. One proceeds by transfinite induction, and the resulting set $\Lambda$ is a set of ordinal numbers. One first proceeds by induction on ordinals $\lambda$, the induction hypothesis being that for $n<\lambda$, 
the following holds: for each $k<n$, the sets $I_{k}, J_{k}$ are defined, so that

$$
\bigoplus_{k<n}\left(\bigoplus_{i \in I_{k}} A_{i}\right)=\bigoplus_{k<n}\left(\bigoplus_{j \in J_{k}} B_{i}\right)
$$

and the sets $I_{k}, J_{k}$ are disjoint, countable subsets of $I$ and $J$ respectively. We conclude that there is an ordinal $\lambda$ such that $I$ is the union of the sets $I_{k}$ for $k<\lambda$, and $J$ is the union of sets $J_{k}$ for $k<\lambda$. For any $k<\lambda$, we apply the induction formula for $n=k$ and $n=k+1$ and obtain

$$
\bigoplus_{i \in I_{k}} A_{i} \cong \bigoplus_{j \in J_{k}} B_{j}
$$

as desired, since both are complementary summads to $\bigoplus_{m<k}\left(\bigoplus_{i \in I_{k}} A_{i}\right)$ in $\bigoplus_{m<k+1}\left(\bigoplus_{i \in I_{k}} A_{i}\right)$.

Theorem 6. If $M$ is an object in a reasonable Abelian category and $M$ is the direct sum of countably small subobjects, then any two direct sum decompositions of $M$ into summands having the exchange property have isomorphic refinements.

Proof. By Lemma 4, any decomposition refines into one in which the summands are countably small. Since a summand of an object with the exchange property again has the exchange property, one may assume that all summands involved are countably small. By Lemma 5, we may then assume that the index set is countable, and in this case the result follows from Theorem 5 .

TheOREM 7. [1, Th. 7.1]. Let $M$ be an object in a reasonable Abelian category which is the direct sum of $\sigma$-small subobjects having the exchange property. Then any two direct sum decompositions of $M$ have isomorphic refinements.

Proof. By Theorem 6, it suffices to show that if $N$ is a summand of $M$, then $N$ is also a direct sum of $\sigma$-small subobjects having the exchange property. By Lemma 5 , it suffices to prove this in the case where $M=\bigoplus_{i=1}^{\infty} A_{i}$ and each $A_{i}$ is $\sigma$-small, in which case $N$ is also $\sigma$-small. We can therefore find subobjects $S_{i}(i=0,1, \cdots)$ of $N$ with $S_{i}$ small, $S_{0}=0, S_{i+1} \supseteqq S_{i}$, and such that $N$ is the union of the $S_{i}$. We proceed by induction on $k$, choosing for each $k$ a subobject $N_{k}$, beginning with $N_{0}=0$. We assume by induction that the $N_{i}$ are independent, that $\bigoplus_{i=1}^{k} N_{i}$ is a summand of $N$, that $S_{k} \subseteq \bigoplus_{i=1}^{k} N_{i}$, and that each $N_{k}$ has the exchange property. Clearly if we can carry out this construction, the theorem is proved, since $N=\bigoplus_{1=i}^{\infty} N_{i}$.

By the exchange property for $\bigoplus_{i=1}^{k-1} N_{i}$, there are submodules 
$A_{i}^{\prime} \subseteq A_{i}$ such that

$$
M=\left(\bigoplus_{i=1}^{k-1} N_{i}\right) \oplus\left(\bigoplus_{i=1}^{\infty} A_{i}^{\prime}\right)
$$

Choose $n(k)$ such that

$$
S_{k} \sqsubseteq\left(\bigoplus_{i=1}^{k-1} N_{i}\right) \oplus\left(\bigoplus_{i=1}^{n \cdot h} A_{i}^{\prime}\right)
$$

Applying the exchange property to the object on the right, assuming that $M=N \oplus B$, we obtain complementary subobjects $N_{k}^{*} \subseteq N, B_{k} \subseteq B$. Let $N^{\prime}$ be the intersection of $N$ with

$$
\left(\bigoplus_{i=1}^{k-1} N_{i}\right) \oplus\left(\bigoplus_{i=1}^{n(k)} A_{i}^{\prime}\right) \oplus B_{i} .
$$

Clearly, $S_{k} \subseteq N_{k}^{\prime}$. Let $N_{k}$ be a complement to $\bigoplus_{i=1}^{k-1} N_{i}$ in $N_{k}^{\prime}$. Since $N_{k}$ is isomorphic to a summand of $\bigoplus_{i=1}^{n(k)} A_{i}^{\prime}, N_{k}$ has the exchange property, and since $N=N_{k}^{\prime} \oplus N_{k}^{*}$, the induction is completed.

Corollary 7.1. Let $M$ be an $R$-module which is a direct sum of countably generated injective modules. Then any summand of $M$ is a direct sum of injective modules and any two direct sum decompositions of $M$ have isomorphic refinements.

In the case where $M$ is a direct sum of countably generated indecomposable injective modules, this is contained in results of Faith and Walker [2].

To give another example, we return to our earlier remark that the above proofs are valid in any full subcategory of a reasonable Abelian category which is closed under summands and direct sums. We apply this to the category of torsion-free reduced modules over an integral domain.

CoRollary 7.2. If a reduced torsion-fiee module $M$ over an integral domain is a direct sum of $R D$-injective modules, then any two direct sum decompositions of $M$ have isomorphic refinements.

Proof. It is clear that a torsion-free $R D$-injective module is algebraically compact in the sense of [11]. Algebraically compact modules have very strong completeness properties which make it easy to check that a reduced torsion-free $R D$-injective module is small in the category of reduced torsion-free modules. The result will follow if we can prove the exchage property for such modules. By [1, Th. 8.2] (or by an elementary argument) we may assume that all of the summands involved are torsion-free and reduced, and using the smallness of the $R D$-injective modules, we may assume that the total number of summands involved is finite. Since the operation of taking $R D$-injective 
envelopes preserves finite direct sums, we may assume that all of the modules involved are $R D$-injective. Using the category isomorphism theorem mentioned in connection with Corollary 4.4, the result now follows since injective modules have the exchange property.

4. Some unsolved problems. It would be nice to weaken or remove the countability requirements in $\S 3$. In particular, it would be nice to weaken the hypotheses of Theorem 7 and make them agree with those of Theorem 6. By analogy with Theorem 1, one might hope to remove all such hypotheses by assuming that the object being decomposed also has the exchange property.

One would like to prove theorems similar to Corollary 4.4 for other classes of modules defined by relative injectivity properties similar to that defining $R D$-injective modules. One theorem in this direction which does not follow from our methods is the classification theorem for complete Abelian groups. A reduced Abelian group is $R D$-injective (or algebraically compact) if and only if it is complete and Hausdorff in its $Z$-adic topology. Any such group is the completion of a direct sum of indecomposable complete groups, and any two such decompositions are isomorphic. The indecomposable complete groups are just the cyclic groups of prime power order and the additive groups of $p$-adic integers. A suitable generalization of the results of $\S 3$ might include similar theorems for modules over other rings.

\section{BIBLIOGRAPHY}

1. P. Crawly and B. Jónsson, Refinements for in finite direct decompositions of algebraic systems, Pacific J. Math. 14 (1964), 797-855.

2. C. Faith and E. Walker, Direct-sum representations of injective modules, J. of Algebra 5 (1967), 203-221.

3. P. Gabriel, Des catégories Abéliennes, Bull. Soc. Math. Fr. 90 (1962), 323-448.

4. P. Gabriel and U. Oberst, Spektralkategorien und reguläre Ringe im von-Neumannschen Sinn, Math. Zeit. 92 (1966), 389-395.

5. I. Kaplansky, Projective modules, Ann. Math. 68 (1958), 373-377.

6. S. MacLane, Homology, Berlin, Springer, 1963.

7. E. Matlis, Injective modules over Noetherian rings, Pacific J. Math. 8 (1958), 511-528.

8. _ـ, Injective modules over Prüfer rings, Nagoya Math. J. 15 (1959), 57-69.

9. $\longrightarrow$, Cotorsion modules, Memoirs Amer. Math. Soc. 49 (1964).

10. C. Nǎstǎsescu and N. Popescu, Sur la structure des objects de certaines catégories, abeliennes, C. R. Acad. Sci. Paris 262 (1966), 1295-1297.

11. R. B. Warfield, Jr., Purity and algebraic compactness for modules, Pacific J. Math. 28 (1969), 699-719.

12. Lelatively injective modules, (to appear).

Received May 6, 1969.

UNIVERSITY OF WASHINGTON 


\section{PACIFIC JOURNAL OF MATHEMATICS}

\section{EDITORS}

H. ROYDEN

Stanford University

Stanford, California

Richard Pierce

University of Washington

Seattle, Washington 98105
J. DUGUNDJI

Department of Mathematics

University of Southern California

Los Angeles, California 90007

BASIL GORDON

University of California

Los Angeles, California 90024

\section{ASSOCIATE EDITORS}
E. F. BECKENBACH
B. H. NEUMANN
F. WOLF
K. YosHIDA

\section{SUPPORTING INSTITUTIONS}

UNIVERSITY OF BRITISH COLUMBIA

CALIFORNIA INSTITUTE OF TECHNOLOGY

UNIVERSITY OF CALIFORNIA

MONTANA STATE UNIVERSITY

UNIVERSITY OF NEVADA

NEW MEXICO STATE UNIVERSITY

OREGON STATE UNIVERSITY

UNIVERSITY OF OREGON

OSAKA UNIVERSITY

UNIVERSITY OF SOUTHERN CALIFORNIA
STANFORD UNIVERSITY

UNIVERSITY OF TOKYO

UNIVERSITY OF UTAH

WASHINGTON STATE UNIVERSITY

UNIVERSITY OF WASHINGTON

*

AMERICAN MATHEMATICAL SOCIETY CHEVRON RESEARCH CORPORATION TRW SYSTEMS

NAVAL WEAPONS CENTER 


\section{Pacific Journal of Mathematics}

\section{Vol. 31, No. $1 \quad$ November, 1969}

James Burton Ax, Injective endomorphisms of varieties and schemes........

Richard Hindman Bouldin, A generalization of the Weinstein-Aronszajn

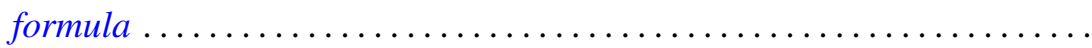

John Martin Chadam, The asymptotic behavior of the Klein-Gordon equation with external potential. II ...............................

Rina Hadass, On the zeros of the solutions of the differential equation

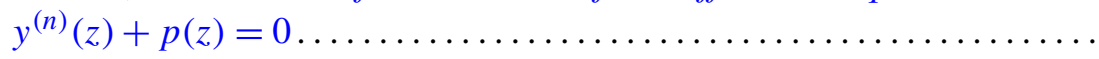

John Sollion Hsia, Integral equivalence of vectors over local modular lattices. II .............................................

Robert Hughes, Boundary behavior of random valued heat polynomial

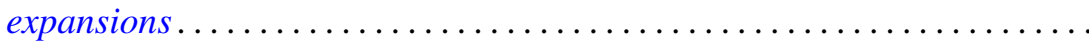

Surender Kumar Jain, Saad H. Mohamed and Surjeet Singh, Rings in which every right ideal is quasi-injective .........................

T. Kawata, On the inversion formula for the characteristic function .........

Erwin Kleinfeld, On right alternative rings without proper right ideals......

Robert Leroy Kruse and David Thomas Price, On the subring structure of

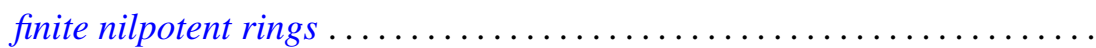

Marvin David Marcus and Stephen J. Pierce, Symmetric positive definite multilinear functionals with a given automorphism. .

William Schumacher Massey, Pontryagin squares in the Thom space of a bundle...

William Schumacher Massey, Proof of a conjecture of Whitney ...

John William Neuberger, Existence of a spectrum for nonlinear transformations

Stephen E. Newman, Measure algebras on idempotent semigroups ...

$\mathrm{K}$. Chandrasekhara Rao, Matrix transformations of some sequence spaces

Robert Bruce Schneider, Some theorems in Fourier analysis on symmetric

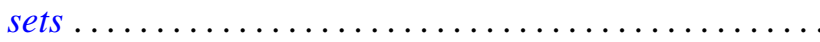

Ulrich F. K. Schoenwaelder, Centralizers of abelian, normal subgroups of hypercyclic groups...

Jerrold Norman Siegel, $G$-spaces, $H$-spaces and $W$-spaces

Robert Irving Soare, Cohesive sets and recursively enumerable Dedekind cuts...

Kwok-Wai Tam, Isometries of certain function spaces .... . .

Awadhesh Kumar Tiwary, Injective hulls of semi-simple modules over regular rings ....

Eldon Jon Vought, Concerning continua not separated by any nonaposyndetic subcontinuum .................... 